\title{
Digital Watermarking of Video using DWT, PCA and Arnold Scrambling Technique applied on a Binary Watermark
}

\author{
Temshichang Jongko ${ }^{1}$, Jeebananda Panda ${ }^{2}$, Asok Bhattacharyya ${ }^{3}$ \\ Department of E\&C Engineering, Delhi Technological University, New Delhi, India ${ }^{1}$ \\ Associate Professor, Department of E\&C Engineering, Delhi Technological University, New Delhi, India ${ }^{2}$ \\ Professor, Department of E\&C Engineering, Delhi Technological University, New Delhi, India ${ }^{3}$
}

\begin{abstract}
In this paper analysis have been performed for a good trade-off between the perceptual quality of the video after watermarking and the robustness of the video against various attacks by embedding the binary watermark into the video frame. The embedding and the extraction of watermark is done in the high frequency domain of Discrete Wavelet Transform since small modifications done in this domain are not perceived by human eye. Arnold Cat Map Scrambling technique is used in the algorithm, which is applied on the binary watermark before embedding to provide some level of security. In the paper four watermarking algorithms are presented to do a comparative study. The normalized correlation and bit error is used to compute the measure of the extracted watermark. The experimental results shows high imperceptibility and high robustness against several attacks especially noisy attacks and filtering attacks in all the cases. The comparison has been shown in the result section.
\end{abstract}

Keywords: Digital Video Watermarking, Discrete Wavelet Transform, Principal Component Analysis, Arnold Cat Map.

\section{INTRODUCTION}

In recent years, the advancement in the digital multimedia technologies has brought many facilities in reproduction, transmission and data manipulation. However this advancement has also brought the problem such as copyright issues. For copyright protection of digital multimedia, watermarking [1] has been proposed. A signal (watermark) is embedded into the data which will indicate whether the content is copyrighted or not. Most of digital video watermarking schemes are based on the techniques of image watermarking. Video watermarking [2] requires specific approaches as it is characterized by temporal and inter frame characteristics. There are two categories for embedding the watermark [3] in a video frame based on the domain which is used for hiding the watermark bits namely the spatial domain and the transform domain. The first one is the spatial domain watermarking where embedding and detection is performed by directly manipulating the pixel intensity value of the video frame. Least significant bit (LSB) is the simplest technique in the spatial domain techniques [4] which directly modifies the intensities of some selected pixels. The second category is the transform domain watermarking, in which the watermark is embedded by changing the frequency components of the video frame. The transformation adopted may be Discrete Cosine transform (DCT), Discrete Fourier Transform (DFT) and Discrete Wavelet transform (DWT) etc. After Applying transformation, the watermark is embedded in the transformed coefficients such that watermark is not visible. The transform domain watermarking scheme are relatively more robust than the spatial domain watermarking particularly in lossy compression, noise addition, pixel removal, cropping, rescaling and rotation

Discrete wavelet transform (DWT) is computationally more efficient than other transforms like DFT and DCT because of its excellent time-frequency localization properties, DWT is very suitable to identify areas in the host video frames where watermark can be embedded imperceptibility. It is known that there exists some amount of correlation between wavelet coefficients even after the decomposition of the video frame.

Wang et al. [5] adopt a key dependent wavelet transform. To take advantage of the localisation and multi resolution property of the wavelet transform. Tao et al. [6] put forward a discrete wavelet based multiple watermarking algorithm, where to improve the robustness the watermark is embedded on the LL and HH sub bands. Wang and Lin [7] proposed a tree based watermarking algorithm. Lou et al. [8] introduce an integer wavelet to protect the copyright of digital data by utilizing encryption technique to enhance the security.

Yung et al. [9] proposed a multiple logo watermarking scheme by on integer wavelet. Where the watermark is permuted using the Arnold transform and is embedded by modifying the coefficients of the HH and LL sub bands. Lin et al.[10] proposed a DWT based blind watermarking scheme by scrambling the watermark using the chaos sequence. Jeebananda Panda et al.[11] proposed a hybrid watermarking scheme based on integer wavelet and Singular value decomposition, where watermark is embedded after performing a chaotic scrambling. 
Principal component analysis (PCA) has the property of transform a number of correlated variables into removing the correlation among the data and is used to uncorrelated variables called the principal components. To hybridize the algorithm i.e. wavelet coefficients, which obtain the Principal components of matrix I, firstly helps in distributing the watermark bits over the sub-bands calculate the covariance matrix used for embedding thus resulting in robust watermarking scheme which is robust to almost all attacks. The PCA can be used in different ways in image and video watermarking methods. Here we have presented a new approach that incorporates wavelet transformation, principal component analysis and Arnold cat map scrambling technique. It was found that the proposed technique is robust to a wide range of attacks.

$$
\mathrm{C}_{\mathrm{I}}=\mathrm{E}\left\{(\mathrm{I}-\mathrm{m}) \times(\mathrm{I}-\mathrm{m})^{\mathrm{T}}\right\}
$$

Where $\mathrm{E}, \mathrm{m}$ and $\mathrm{T}$ denote expectation operation, mean of matrix $\mathrm{X}$ and matrix transpose, respectively. The principal components of $\mathrm{X}$ are the eigenvectors of $\mathrm{C}_{\mathrm{I}}$ which is obtain as

$$
\mathrm{C}_{\mathrm{I}} \Phi=\lambda \Phi
$$

Section II presents the proposed watermarking method and Where $\Phi$ and $\lambda$ are the matrices of the eigenvectors and Section III shows experimental results of the proposed the eigen values defined as $\Phi=\left(\mathrm{e}_{1}, \mathrm{e}_{2}, \mathrm{e}_{3}, \ldots, \mathrm{e}_{\mathrm{n}}\right)$ and method. Conclusion is presented in Section IV.

\section{RELATED BACKGROUND}

This section briefly describes the techniques and methods that have been used by the watermarking schemes in the paper, namely. The discrete wavelet transform, Principal component analysis and the Arnold transform.

\section{A. Discrete Wavelet Transform}

The DWT is commonly used in signal processing applications. 2D Discrete Wavelet Transform (DWT) decomposes a video frames into sub images, 3 details and 1 approximation. The approximation sub images is lower resolution approximation image (LL) however the details sub images are horizontal (HL), vertical (LH) and diagonal (HH) detail components. The main advantage of wavelet transform is its compatibility with the model aspect of the Human Visual System (HVS) as compared to FFT or DCT. In general most of the image energy is concentrated at the lower frequency coefficient sets LL and therefore embedding watermarks in these coefficient sets may degrade the image significantly. Embedding in the low frequency coefficient sets, however, could increase robustness significantly. On the other hand, the high frequency coefficient sets $\mathrm{HH}$ include the edges and textures of the image and the human eye is not generally sensitive to changes in such coefficient sets. This allows the watermark to be embedded without being perceived by the human eye. For a one level decomposition, the two dimensional discrete wavelet transform of the image function $\mathrm{f}(\mathrm{x}, \mathrm{y})$ can be written as

$$
\begin{aligned}
& \mathrm{LL}=[(\mathrm{f}(\mathrm{x}, \mathrm{y}) * \phi(-\mathrm{x}) \phi(-\mathrm{y}))(2 \mathrm{n}, 2 \mathrm{~m})]_{(\mathrm{n}, \mathrm{m}) \in \mathrm{Z}^{2}} \\
& \mathrm{LH}=[(\mathrm{f}(\mathrm{x}, \mathrm{y}) * \phi(-\mathrm{x}) \psi(-\mathrm{y}))(2 \mathrm{n}, 2 \mathrm{~m})]_{(\mathrm{n}, \mathrm{m}) \in \mathrm{Z}^{2}} \\
& \mathrm{HL}=[(\mathrm{f}(\mathrm{x}, \mathrm{y}) * \psi(-\mathrm{x}) \phi(-\mathrm{y}))(2 \mathrm{n}, 2 \mathrm{~m})]_{(\mathrm{n}, \mathrm{m}) \in \mathrm{Z}^{2}} \\
& \quad \mathrm{HH} \\
& \quad=[(\mathrm{f}(\mathrm{x}, \mathrm{y}) * \phi(-\mathrm{x}) \phi(-\mathrm{y}))(2 \mathrm{n}, 2 \mathrm{~m})]_{(\mathrm{n}, \mathrm{m}) \in \mathrm{Z}^{2}}
\end{aligned}
$$

\section{B. Principal Component Analysis}

Principal component analysis (PCA) is a mathematical procedure that is concerned with data reduction and interpretation by explaining the variance-covariance structure of the data with the help of linear combination of the original variables. Moreover, principal component analysis can also be thought as a mathematical tool that

$\lambda=\left(\lambda_{1}, \lambda_{2}, \lambda_{3}, \ldots, \lambda_{n}\right)$. The matrix $\Phi$ is an orthogonal matrix called basis function of PCA. Principal component analysis transforms the correlated image into uncorrelated coefficients by taking the inner product of the image with basis function denoted by $\Phi$

$$
\mathrm{Y}=\Phi^{T} I
$$

Where $\mathrm{Y}$ is the $\mathrm{PC}$ matrix which represents the principle component of matrix I.

\section{Arnold Cat Map}

Two dimensional Arnold cat map shuffles the pixels positions of the original image without changing the pixels gray level intensities. The $2 \mathrm{D}$ Arnold cat map can be obtained by

$$
\begin{gathered}
X^{\prime}=[X+p Y] \bmod (n) \\
Y^{\prime}=[q X+(p q+1) Y] \bmod (n)
\end{gathered}
$$

Here, $\left(X^{\prime}, Y^{\prime}\right)$ represents the new position of $(X, Y)$ after Arnold's cat transform and $\mathrm{n}$ is the dimension of the image. $p$ and $q$ are the initial values in which the sensitivity of cat map depends. Therefore $p$ and $q$ can be used as keys.

The main aim for using the Arnold transform is to provide security to the watermarking scheme, by scrambling the watermark before embedding.

\section{Binary watermark}

The advantages of binary watermark over the colour watermark are that there is a lot of complexity in embedding a value for each intensity value for a colour watermark or a gray scale watermark. At the same time in the extraction process it is difficult to get a true colour value.

\section{III.THE ALGORITHMS USED}

In this section different algorithms are presented which were performed in order to do a comparative study to bring out the proposed method.

A. Scrambled watermark embedded on the principal components of the wavelet coefficients

In this algorithm the host video frame is decomposed to wavelet coefficients using 3 level DWT from there the principal component is calculated and the scrambled watermark which is scrambled watermark using Arnold 
transformism embedded on the principal component. The Step2. From each watermarked frame the RGB colour detailed algorithm for embedding and extraction watermark is discussed in the following

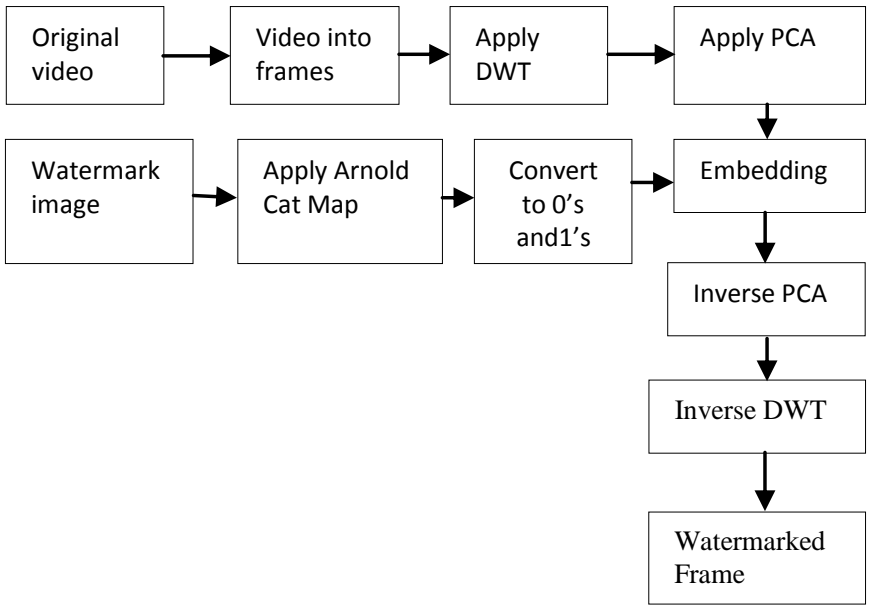

Fig.1. Watermark embedding algorithm for watermarking scheme where scrambled watermark is applied on Principal components of the Wavelet coefficients.

Watermark Embedding

Step1. The Host video is read and is divided into video frames.

Step2. From each frame the RGB components is separated and from there the red component is selected for embedding the watermark.

Step3. A 3 level DWT is performed on the red color component of the video frame. The diagonal $\left(\mathrm{HH}_{34}\right)$ detailed component of the wavelet transform is selected to embedding the watermark.

Step4. The diagonal $\left(\mathrm{HH}_{34}\right)$ detailed component is divided into non-overlapping blocks and PCA is applied to obtain the Principal components for embedding the watermark.

Step5. The binary watermark image BW is read and Arnold Cat Map is performing on the watermark image. The initial value and the number of iterations is hold as the security for the proposed watermarking algorithm.

Step6. Convert the scrambled binary watermark into a vector of $\mathrm{BW}$ ' $=\left\{\mathrm{bw}_{1}{ }^{\prime}, \mathrm{bw}_{2},{ }^{\prime}, \ldots, \mathrm{bw} \mathrm{m}_{\mathrm{n}}\right.$ ' $\}$ of ' 0 's and'1's.Step7. The watermark is embedded into the Principal components PCB of each non-overlapping block using the following Equation.

$$
\mathrm{PCB}^{\prime}(\mathrm{m}, \mathrm{n})=\mathrm{PCB}(\mathrm{m}, \mathrm{n})+\mathrm{k} \cdot \mathrm{w}(\mathrm{m}, \mathrm{n})
$$

Where, $\mathrm{k}$ is the embedding strength. The PCB' and PCB are the modified principal components and the original principal components.

Step8. Apply inverse PCA on the modified Principal components of the $\mathrm{HH}_{34}$ band to obtain the modified wavelet coefficients.

Step9. Apply inverse DWT to obtain the Red component of the frame. Then reconstruct the watermarked frame $X_{\mathrm{w}}$.

\section{Watermark Extraction}

Step1. Read the watermarked video and divide it into video frames.
Step2. From each watermarked frame the RGB colour
component is separated and from there component is selected for extracting the watermark.

Step3. Apply 3 levels DWT on the red component of the watermarked video frame. The multi-level DWT is applied to obtain $\mathrm{HH}_{34}$ detailed component as same as in the embedding procedure.

Step4. Divide the sub band $\mathrm{HH}_{34}$ into non overlapping block.

Step5. Block based PCA is applied on each non overlapping block of $\mathrm{HH}_{34}$ subband to obtain the watermarked Principal components PCB'.

Step6. The watermark is extracted from each nonoverlapping block using the following equation

$$
\mathrm{BW}^{\prime}=\left(\mathrm{PCB}^{\prime}-\mathrm{PCB}\right) / \mathrm{k} \text {. }
$$

Where $\mathrm{PCB}$ ' and $\mathrm{PCB}$ are the principal components of watermarked and original video frame and $\mathrm{k}$ is the embedding strength.

Step7. Apply Arnold Cat map on the extracted watermark and apply thresholding to obtain the embedded watermark image.

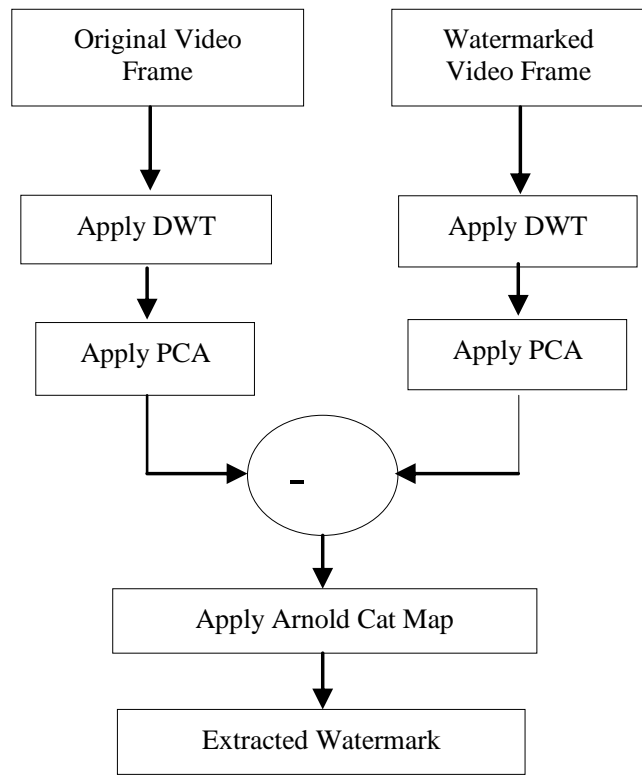

Fig.2. Watermark Extraction Algorithm for watermarking scheme where scrambled watermark is applied on Principal components of the Wavelet coefficients.

B. Scrambled watermark embedded on the wavelet coefficient

In this the host video frame is decomposed using three 3 level DWT and the scramble watermark obtain from Arnold transform is embedded on the wavelet coefficients. The algorithm for embedding and the extraction watermark is discussed in the following.

Embedding

Step1. Read the video frames and select the red component for embedding the watermark.

Step2. Apply 3 levels DWT on the Red component and select $\mathrm{HH}_{34}$ sub band and divide it into non-overlapping blocks. 
Step3. Read the binary watermark image.

Step4. Apply Arnold Cat Map on the binary image and convert the scrambled image into vectors of 0 's and 1's.

Step5. The watermark bits is inserted on each nonoverlapping blocks of the wavelet coefficients as.

$$
\mathrm{cH}^{\prime}=\mathrm{cH}+\mathrm{k} . \mathrm{W}
$$

Where $\mathrm{cH}$ is the wavelet coefficient obtained after apply 3 level DWT, $\mathrm{k}$ is the embedding strength and $\mathrm{W}$ is the watermark bit obtained after scrambling. $\mathrm{cH}^{\prime}$ is the modified wavelet coefficient.

Step6. Apply inverse 3 levels DWT

Step7. Merge colour component to obtain the watermarked frame.

\section{Extraction}

Step1. Read the watermarked Frame and select the Red colour component for extraction.

Step2. Apply 3 level DWT on the Red colour Component and select $\mathrm{HH}_{34}$ sub band and divide it into nonoverlapping blocks.

Step3. The watermark bits is extracted from each nonoverlapping blocks by as

$$
\mathrm{W}^{\prime}=\left(\mathrm{cH}_{\mathrm{w}}-\mathrm{cH}\right) / \mathrm{k} \text {. }
$$

Where $\mathrm{cH}_{\mathrm{w}}$ is the discrete wavelet coefficient obtained after applying 3 levels DWT. And $\mathrm{cH}$ is the Discrete Wavelet coefficient of the Host video frame.

Step4. Convert back to binary image and apply Arnold Cat map to the extracted watermark bits to obtain the watermark image.

C. Principal components of the scrambled watermark embedded on the wavelet coefficients

In this, the host video frame is decomposed using 3 level DWT and the principal components of the scrambled watermark is embedded on the obtained wavelet coefficients. The detailed embedding and extraction algorithm is discussed in the following

Embedding

Step1. Read the video frame and select Red colour component for embedding.

Step2. Apply 3 levels DWT to the Red component, select $\mathrm{HH}_{34}$ sub band and divide it into non-overlapping blocks.

Step3. Read the binary Watermark.

Step4. Apply Arnold Cat map to watermark image and convert it into 0's and 1's.

Step5. Apply PCA on the scrambled watermark bits.

Step6. The watermark bits is embedded into each nonoverlapping blocks of the wavelet coefficients as

$$
\mathrm{cH}^{\prime}=\mathrm{cH}+\alpha \mathrm{k} . \mathrm{W}_{\mathrm{pca}}
$$

where $\mathrm{cH}$ is the wavelet coefficient after 3 level DWT, $\mathrm{W}_{\mathrm{pca}}$ is the principal components after applying PCA in scrambled watermark bits and $\alpha \mathrm{k}$ is the embedding strength.

Step7. Apply inverse 3 levels DWT

Step8. Merge colour to obtain the watermarked frame.

\section{Extraction}

Step1. Read the watermarked frame and select the red colour component for watermark extraction.

Step2. Apply 3 levels DWT on the red colour component, select the $\mathrm{HH}_{34}$ sub band and divide it into nonoverlapping blocks.

Step3. The watermark bits is extracted from each nonoverlapping blocks using the equation as

$$
W_{P c a}^{\prime}=\left(\mathrm{cH}_{\mathrm{w}}-\mathrm{cH}\right) / \mathrm{k} \text {. }
$$

Where $\mathrm{cH}_{\mathrm{w}}$ is the coefficient obtains after DWT on the watermarked frame and $\mathrm{cH}$ is the coefficient obtain after WT on the original video frame.

Step4. Apply inverse PCA.

Step5. Convert the extracted watermark into binary image. Step6. Apply Arnold cat map to obtain the watermark image.

D. The principal components of the scrambled watermark is embedded on the principal components of the wavelet coefficients

In this, the host video frame is decomposed using 3 levels DWT and the principal components of the wavelet coefficients is calculated. The principal components of the scrambled watermark are embedded on the principal components of the wavelet coefficients. The detail embedding and the extraction algorithm is discussed in the following.

Embedding

Step1. Read the Video Frames and select red colour component for watermark insertion.

Step2. Apply 3 levels DWT on the Red component and select $\mathrm{HH}_{34}$ sub-band and divide it into non-overlapping blocks.

Step3. Apply based PCA on the non-overlapping blocks.

Step4. Read the binary watermark.

Step5. Apply Arnold Cat Map on the watermark image and convert it to vectors of 0's and 1's.

Step6. Apply PCA on the scrambled watermark image.

Step7. The watermark bits are inserted on each nonoverlapping block using the equation

$$
\mathrm{PCA}^{\prime}=\mathrm{PCA}+\mathrm{k} \cdot \mathrm{W}_{\mathrm{pca}}
$$

Where PCA' is the modified Principal components and PCA is the principal components obtain after applying PCA on the $\mathrm{HH}_{34}$ sub-band. $\mathrm{k}$ is the embedding strength.

Step8. Apply inverse PCA on the modified Principal components.

Step9. Apply inverse DWT on the obtained.

Step10. Merge the colour component to obtain the watermarked frame.

Extraction

Step1. Read the watermarked video frame and select the red colour component for watermark extraction.

Step2. Apply 3 levels DWT on the red colour component and select the $\mathrm{HH}_{34}$ sub band and divide it into nonoverlapping blocks.

Step3. Apply PCA on the non-overlapping blocks to obtain the principal component PCAw. 
Step4. The watermark bit are extracted from each non- Where $\mathrm{W}$ and $\mathrm{W}$ ' are the original and watermark image. overlapping using the equation

$$
W_{P c a}^{\prime} W_{P c a}^{\prime}=\left(\mathrm{PCA}_{\mathrm{w}}-\mathrm{PCA}\right) / \mathrm{k}
$$

To measure the robustness of the watermarking scheme some attacks are applied to the watermarked frame.

Where $\mathrm{PCA}_{\mathrm{w}}$ is the Principal component obtained after applying PCA on the watermarked frame. And PCA is the Principal components of the original Video frame.

Step5. Apply inverse PCA on the extracted watermark bits.

Step6. Convert the bits into binary image and apply Arnold Cat map to obtain the watermark image to obtain the extracted watermark.

\section{IV.EXPERIMENTAL RESULTS}

For the experimental analysis sample video of sequence 'Xylophone.avi' of dimension $512 \times 512$ and binary watermark logo 'apple.gif' of size $32 \times 32$ is used for all the algorithms. For all the algorithms both the $\mathrm{p}$ and $\mathrm{q}$ value for Arnold cat map scrambling function is chosen to be 1. The embedding strength for the entire algorithm is set to 22. Fig3. and Fig4. Shows the original video frame and the watermark in the experiment.

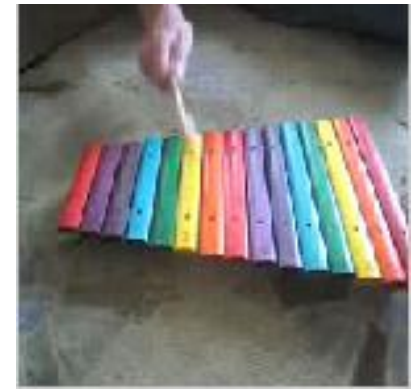

Fig.3. Original Frame

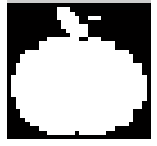

Fig.4. Original watermark

For evaluating the perceptual quality of the watermarking schemes, Peak Signal to Noise Ratio (PSNR) is used which is given by

$$
\begin{aligned}
& \text { PSNR }=10 \log \frac{256^{2}}{\mathrm{MSE}} \\
& \operatorname{MSE}=\sum_{\mathrm{i}=1}^{\mathrm{M}} \sum_{\mathrm{j}=1}^{\mathrm{N}}\left[\mathrm{I}(\mathrm{i}, \mathrm{j})-\mathrm{I}^{\prime}(\mathrm{i}, \mathrm{j})\right]^{2}
\end{aligned}
$$

Where $\mathrm{M}, \mathrm{N}$ are the size of the frame. I, I' are the original and watermarked video frame.

Also the Bit error is used to measure the robustness the algorithm

$$
\mathrm{BER}=\frac{\sum_{\mathrm{i}=1}^{\mathrm{M}} \sum_{\mathrm{j}=1}^{\mathrm{N}} \mathrm{W}(\mathrm{i}, \mathrm{j}) \oplus \mathrm{W}^{\prime}(\mathrm{i}, \mathrm{j})}{\mathrm{M} \cdot \mathrm{N}}
$$

Where $\mathrm{W}$ and $\mathrm{W}^{\prime}$ is the original and extracted watermark. Normalised cross-correlation method is also used to measure similarity between the watermark and the extracted watermark

$$
\mathrm{NC}=\frac{\sum_{\mathrm{i}} \sum_{\mathrm{j}} \mathrm{W}(\mathrm{i}, \mathrm{j}) \mathrm{W}^{\prime}(\mathrm{i}, \mathrm{j})}{\sqrt{\sum_{\mathrm{i}} \sum_{\mathrm{j}} \mathrm{W}(\mathrm{i}, \mathrm{j}) \sum_{\mathrm{i}} \sum_{\mathrm{j}} \mathrm{W}(\mathrm{i}, \mathrm{j})}}
$$

The attack analysis of the watermarking schemes is as shown below.

Algorithm $\mathrm{A} \rightarrow$ Scrambled watermark embedded on the principal components of the wavelet coefficients.

Algorithm B $\rightarrow$ Scrambled watermark embedded on the wavelet coefficient.

Algorithm $\mathrm{C} \rightarrow$ Principal Components of the scrambled

\begin{tabular}{|c|c|c|c|c|}
\hline \multirow[t]{2}{*}{ Attacks } & \multicolumn{4}{|l|}{ Bit error } \\
\hline & $\begin{array}{l}\text { Algorith } \\
\text { m A }\end{array}$ & $\begin{array}{l}\text { Algorith } \\
\text { m B }\end{array}$ & $\begin{array}{l}\text { Algorith } \\
\mathrm{m} \mathrm{C}\end{array}$ & $\begin{array}{l}\text { Algorith } \\
\text { m D }\end{array}$ \\
\hline $\begin{array}{l}\text { Salt\&pepper } \\
\text { noise }(0.001)\end{array}$ & 0.0079 & 0.0107 & 0.0225 & 0.0098 \\
\hline $\begin{array}{l}\text { Salt\&pepper } \\
\text { noise }(0.01)\end{array}$ & 0.0615 & 0.1250 & 0.2063 & 0.1025 \\
\hline $\begin{array}{l}\text { Poisson } \\
\text { noise }\end{array}$ & 0.0225 & 0.2432 & 0.2280 & 0.0586 \\
\hline $\begin{array}{l}\text { Speckle } \\
\text { noise }(0.001)\end{array}$ & 0.0137 & 0.7627 & 0.0371 & 0.0291 \\
\hline $\begin{array}{l}\text { Speckle } \\
\text { Noise }(0.01)\end{array}$ & 0.0332 & 0.2539 & 0.2329 & 0.0732 \\
\hline $\begin{array}{l}\text { Gaussian } \\
\text { noise }(0.001)\end{array}$ & 0.0049 & 0.2510 & 0.1672 & 0.0273 \\
\hline $\begin{array}{l}\text { Gaussian } \\
\text { noise }(0.01)\end{array}$ & 0.1877 & 0.3145 & 0.3936 & 0.2471 \\
\hline $\begin{array}{l}\text { Gaussian } \\
\text { Filter[2 2] }\end{array}$ & 0.0303 & 0.1094 & 0.1028 & 0.0488 \\
\hline
\end{tabular}
watermark embedded on the wavelet coefficients.

\begin{tabular}{|c|c|c|c|c|}
\hline \multirow{2}{*}{ Attacks } & \multicolumn{4}{|l|}{ Bit error } \\
\hline & $\begin{array}{l}\text { Algorith } \\
\mathrm{m} \mathrm{A}\end{array}$ & $\begin{array}{l}\text { Algorith } \\
\text { m B }\end{array}$ & $\begin{array}{l}\text { Algorithm } \\
\text { C }\end{array}$ & $\begin{array}{l}\text { Algorith } \\
\mathrm{m} \mathrm{D}\end{array}$ \\
\hline $\begin{array}{l}\text { Gaussian } \\
\text { Filter[3 } 3 \text { 3] }\end{array}$ & 0.0078 & 0.0527 & 0.0090 & 0.0081 \\
\hline $\begin{array}{l}\text { Gaussian } \\
\text { Filter[5 5] }\end{array}$ & 0.0079 & 0.0547 & 0.0100 & 0.0090 \\
\hline $\begin{array}{l}\text { Median } \\
\text { Filter[2 2] }\end{array}$ & 0.0771 & 0.1063 & 0.1040 & 0.1387 \\
\hline $\begin{array}{l}\text { Median } \\
\text { Filter[3 3] }\end{array}$ & 0.1309 & 0.3398 & 0.1484 & 0.1357 \\
\hline $\begin{array}{l}\text { Median } \\
\text { Filter[5 5] }\end{array}$ & 0.2964 & 0.4287 & 0.3457 & 0.3032 \\
\hline $\begin{array}{l}\text { Mean } \\
\text { Filter[2 2] }\end{array}$ & 0.0303 & 0.1094 & 0.1028 & 0.0488 \\
\hline $\begin{array}{l}\text { Mean } \\
\text { Filter[3 } 3 \text { 3] } \\
\end{array}$ & 0.4075 & 0.4600 & 0.4292 & 0.4277 \\
\hline $\begin{array}{l}\text { Mean } \\
\text { Filter[5 5] }\end{array}$ & 0.4575 & 0.5706 & 0.4707 & 0.4756 \\
\hline $\begin{array}{l}\text { Histo- } \\
\text { equal }\end{array}$ & 0.0527 & 0.2285 & 0.0420 & 0.0031 \\
\hline $\begin{array}{l}\text { Gamma- } \\
\text { correction }\end{array}$ & 0.0020 & 0.2070 & 0.0052 & 0.0003 \\
\hline $\begin{array}{l}\text { Cropping } \\
(10 \%)\end{array}$ & 0.0020 & 0.1709 & 0.2161 & 0.0031 \\
\hline $\begin{array}{l}\text { Cropping } \\
(20 \%)\end{array}$ & 0.0743 & 0.1094 & 0.0859 & 0.8083 \\
\hline $\begin{array}{l}\text { Cropping } \\
(30 \%)\end{array}$ & 0.1495 & 0.3359 & 0.3291 & 0.9801 \\
\hline
\end{tabular}

Algorithm D $\rightarrow$ the principal components of the scrambled watermark is embedded on the principal components of the wavelet coefficients 
Table.1. Comparison between algorithms in terms of bit error

\begin{tabular}{|l|l|l|l|l|}
\hline \multirow{2}{*}{ Attacks } & \multicolumn{4}{|l|}{ Normalised Correlation } \\
\cline { 2 - 5 } & $\begin{array}{l}\text { Algorith } \\
\text { m A }\end{array}$ & $\begin{array}{l}\text { Algorit } \\
\text { hm B }\end{array}$ & $\begin{array}{l}\text { Algorith } \\
\text { m C }\end{array}$ & $\begin{array}{l}\text { Algorith } \\
\text { m D }\end{array}$ \\
\hline $\begin{array}{l}\text { Salt \& pepper } \\
\text { noise(0.001) }\end{array}$ & 0.9927 & 0.9893 & 0.9783 & 0.9901 \\
\hline $\begin{array}{l}\text { Salt \& pepper } \\
\text { noise(0.01) }\end{array}$ & 0.9440 & 0.7345 & 0.8192 & 0.8900 \\
\hline Poisson noise & 0.9754 & 0.8915 & 0.8011 & 0.9494 \\
\hline $\begin{array}{l}\text { Gaussian } \\
\text { noise(0.001) }\end{array}$ & 0.9898 & 0.8021 & 0.8373 & 0.9711 \\
\hline $\begin{array}{l}\text { Gaussian } \\
\text { noise(0.01) }\end{array}$ & 0.8373 & 0.6855 & 0.5895 & 0.7251 \\
\hline $\begin{array}{l}\text { Speckle } \\
\text { noise(0.001) }\end{array}$ & 0.9934 & 0.7627 & 0.9602 & 0.9168 \\
\hline $\begin{array}{l}\text { Gaussian } \\
\text { Filter[2 2] }\end{array}$ & 0.9788 & 0.9500 & 0.9024 & 0.9450 \\
\hline
\end{tabular}

\begin{tabular}{|l|l|l|l|l|}
\hline \multirow{2}{*}{ Attacks } & \multicolumn{4}{|l|}{ Normalised correlation } \\
\cline { 2 - 5 } & $\begin{array}{l}\text { Algorith } \\
\text { m A }\end{array}$ & $\begin{array}{l}\text { Algorith } \\
\text { m B }\end{array}$ & $\begin{array}{l}\text { Algorith } \\
\text { m C }\end{array}$ & $\begin{array}{l}\text { Algorith } \\
\text { m D }\end{array}$ \\
\hline $\begin{array}{l}\text { Gaussian } \\
\text { Filter[3 3] }\end{array}$ & 0.9927 & 0.9473 & 0.9901 & 0.9921 \\
\hline $\begin{array}{l}\text { Gaussian } \\
\text { Filter[5 5] }\end{array}$ & 0.9934 & 0.9453 & 0.9845 & 0.9910 \\
\hline $\begin{array}{l}\text { Mean } \\
\text { Filter[2 2] }\end{array}$ & 0.9788 & 0.7890 & 0.9024 & 0.9458 \\
\hline $\begin{array}{l}\text { Mean } \\
\text { Filter[3 3] }\end{array}$ & 0.6136 & 0.5908 & 0.5696 & 0.5823 \\
\hline $\begin{array}{l}\text { Mean } \\
\text { Filter[5 5] }\end{array}$ & 0.5600 & 0.4600 & 0.4919 & 0.5823 \\
\hline $\begin{array}{l}\text { Median } \\
\text { Filter [2 2] }\end{array}$ & 0.9125 & 0.7634 & 0.8499 & 0.8300 \\
\hline $\begin{array}{l}\text { Median } \\
\text { Filter [3 3] }\end{array}$ & 0.7621 & 0.6602 & 0.7089 & 0.7589 \\
\hline $\begin{array}{l}\text { Median } \\
\text { Filter [5 5] }\end{array}$ & 0.3964 & 0.5713 & 0.6438 & 0.6949 \\
\hline Histo-equal & 0.9024 & 0.7715 & 0.9446 & 0.9538 \\
\hline $\begin{array}{l}\text { Gamma- } \\
\text { correction }\end{array}$ & 0.9982 & 0.7930 & 0.9821 & 0.9993 \\
\hline $\begin{array}{l}\text { Cropping } \\
(10 \%)\end{array}$ & 0.9643 & 0.8402 & 0.8205 & 0.9403 \\
\hline $\begin{array}{l}\text { Cropping } \\
\text { (20\%) }\end{array}$ & 0.9287 & 0.8906 & 0.8879 & 0.9012 \\
\hline $\begin{array}{l}\text { Cropping } \\
\text { (30\%) }\end{array}$ & 0.8503 & 0.6641 & 0.6420 & 0.8083 \\
\hline
\end{tabular}

Table.2. Comparison between Algorithms using Normalised Correlation

From the table it is observed that the Algorithm A i.e. the algorithm where the host video frame is decomposed using 3 level DWT and the scrambled watermark is embedded on the principal component of the wavelet coefficients obtained after decomposing the host video frame gives a better result under attack as compared to the other three algorithm, except for gamma-correction and histogram equalisation attack where the Algorithm 4 i.e. the algorithm where the principal component of the scrambled watermark is embedded on the principal component of the wavelet coefficient obtained after decomposing the host video frame, gives a better result.
For the proposed Algorithm-A, the PSNR value between watermarked and the original video frame without attack is found to be 44.3278 and the Bit error rate of the extracted watermarked is found to be 0.0068 , normalised correlation .9892 .

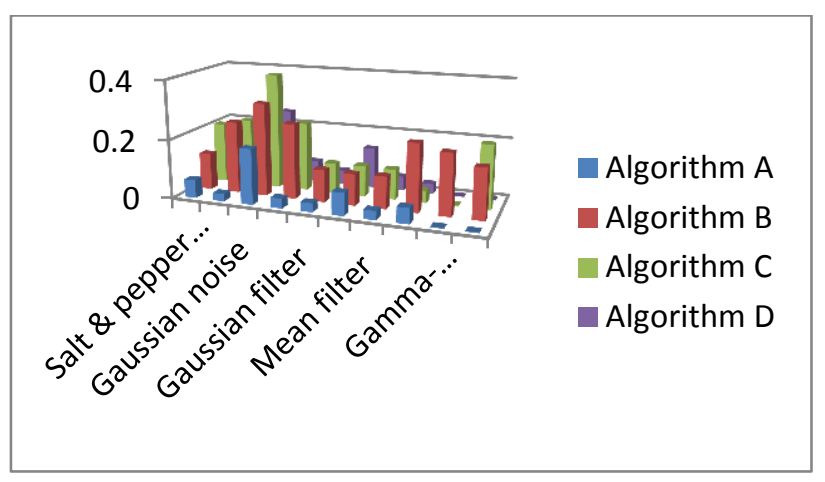

Fig.5. Bar chart for bit error

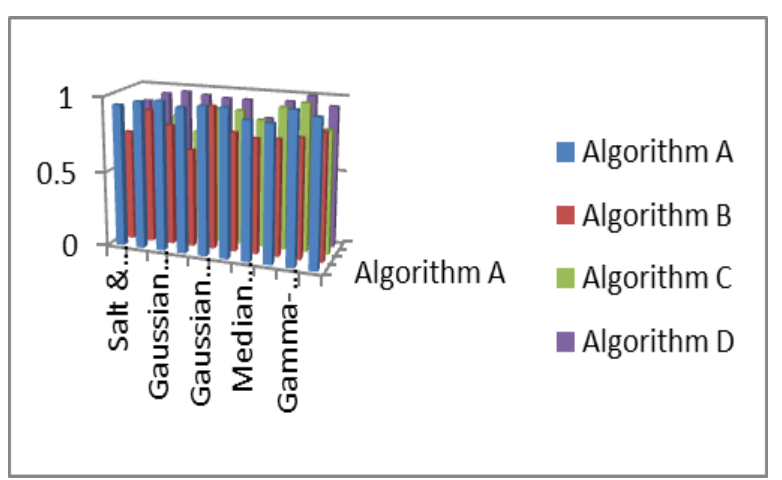

Fig.6. Bar chart for Normalised correlation

Figure below shows some of the watermarked image after attacks and their corresponding extracted watermark.

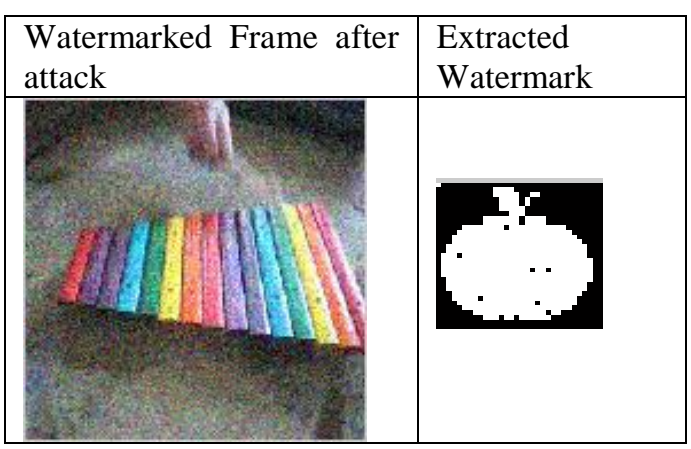

Fig.7. after Gaussian Noise attack

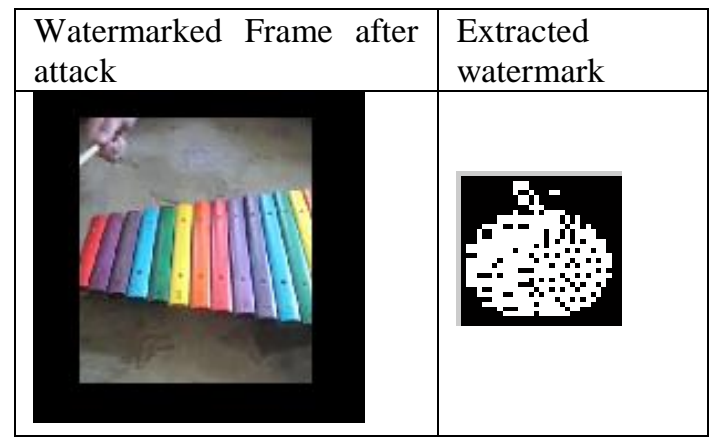

Fig.8. after cropping attack 


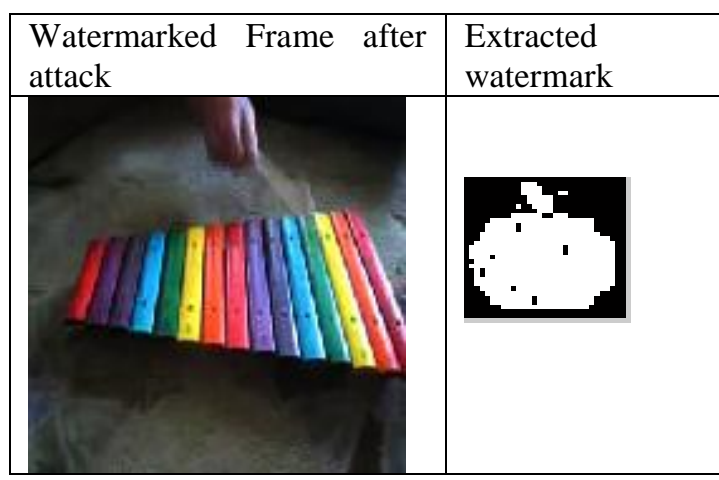

Fig.9. after Gamma Correction attack

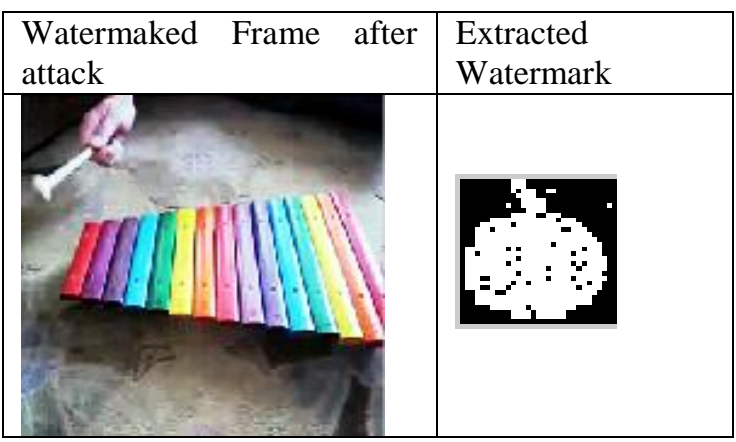

Fig.10. After histogram correction attack

\section{CONCLUSION}

After analysing the performance of all the algorithms after attacks it was found that the proposed watermarking Algorithm-A gives a better performance than the other algorithms. The proposed watermarking method is robust against a wide variety of attacks such as the Gaussian noise, salt and pepper noise attack, speckle noise, Poisson attack, cropping, Gaussian filtering Median filtering, Histogram-equalization and Gamma correction. It is observed all the algorithms could not withstand rotation attack which can be dealt in the future. The proposed watermarking algorithm gives a great level of imperceptibility, which is major requirement for a watermarking technique.

\section{REFERENCES}

[1] H. H. Yu, D. Kundur, and C.-Y. Li. Spies, thieves, and lies: The battle for multimedia in the digital era

[2] J. Dittmann, M. Steinebach, P. Wohlmacher, and R. Ackermann. Digital watermarks enablingE-commerce strategies: conditional and user specific access to services and resources. EURASIP Journal on Applied Signal Processing, 2002(2):174-184, Feb. 2002.

[3] Van Schyndel, R.G., Tirkel, A.Z, and Osborne, C.F, "A digital Watermark." Proc. of the IEEE Int. Conference on Image Processing. Vol. 2, (1994): pp. 86-90.

[4] Parthasarathy A.k, Kak S. "An Improved Method of Content Based Image Watermarking”. IEEE Transaction on broadcasting, Vol.53, no.2, pp.468-479, June 2007.

[5] Wang Y., Doherty J.F, Dyck V.R.E. "A wavelet-based watermarking algorithm for ownership verification of digital images". IEEE Trans. Image process, 11, pp.77-88, 2002.

[6] Tao P., Eskicioglu A.M... "A robust multiple watermarking scheme in the discrete wavelet transform domain". Proceeding of the SPIE, Vol.5601, pp.133-144, 2004.

[7] Wang S.H., Lin Y.P. "Wavelet Tree quantization for copyright protection for watermarking", IEEE Trans. Image Process, pp.154$165,2002$.
[8] Lou Y et al. "Study of digital elevation mode data watermark via integer wavelets." Journal software, 16(6), pp.1096-1103,2005

[9] Yang Y, Huang D, Liu D, "An integer wavelet based multiple logowatermarking schemes". In IEEE, vol.2. pp.175-179,2006

[10] Lin.Q, LiuZ. Feng G. "DWT based on watermarking algorithm and its implementing with DSP”, IEEE Explore, pp.131-134, 2009.

[11] J Panda et.al. Digital watermarking in integer wavelet domain using hybrid technique in Advance in Computer Engineering (ACE), 2010 International Conference on 2010.IEEE 